\title{
Exciton-induced lattice defect formation
}

\author{
E.V. Savchenko and A.N. Ogurtsov \\ B. Verkin Institute for Low Temperature Physics and Engineering \\ of the National Academy of Science of Ukraine, 47 Lenin Ave., Kharkov 61103, Ukraine \\ E-mail: savchenko@ilt.kharkov.ua
}

\section{G. Zimmerer}

Institut für Experimentalphysik der Universität Hamburg, Hamburg 22761, Germany

Received December 25, 2002

\begin{abstract}
The lattice defect formation in solid Ne induced by electronic excitation was studied using the selective vacuum ultraviolet spectroscopy method. The samples were excited with synchrotron radiation in the range of excitonic absorption $n=2 \Gamma(3 / 2)$. The dose dependence of the intensity distribution in the band of atomic type self-trapped exciton luminescence was analyzed. A direct evidence of the formation and accumulation of point lattice defects in solid Ne via the excitonic mechanism was obtained for the first time. The model of the permanent lattice defect formation is discussed.
\end{abstract}

PACS: 61.82.Ms, 71.35.-y, 78.55.Hx

\section{Introduction}

The mechanisms of material modification via electronic subsystem are of considerable interest from the point of view of the fundamental solid-state physics, and their understanding is an important prerequisite for many novel technological applications in material and surface sciences, micro- and nanoelectronics, photochemistry. The basis for the physics of electronically induced lattice rearrangement is a concentration of the excitation energy in the relaxation process within a volume about that of a unit cell followed by the energy transfer to the crystal surrounding. A variety of inelastic processes induced by excitation of the electronic subsystem were studied in wide-gap insulators [1-6] and was found more recently even in narrow-gap materials [7]. The main phenomena under investigation are atomic processes such as lattice defect formation or annealing of the existing defects, mass diffusion, desorption of atoms, ions and molecules from surfaces.

Considerable progress was made towards an insight into their mechanisms. However, many details of the electronically induced processes underlying these phenomena are still not understood in deep. Rare gas solids (RGS) with their simple lattice and well-defined electronic structure are excellent objects for investiga- tion of the electronically induced phenomena. Small binding energies in conjunction with a strong exciton-phonon interaction result in a high quantum yield of electronically induced defect formation and desorption that makes RGS especially suitable for experimental study. Moreover, study of electronically induced atomic processes in the above solids offers unique possibility to elucidate the role of acoustic phonons in these processes. The pronounced effects of exciton self-trapping observed in RGS are essential for efficient energy localization and transfer to induce a lattice modification. It is well known [1-3,8-10] that in solid $\mathrm{Ne}$ the excitons are self-trapped predominantly into atomic type states (A-STE). A rich structure of the atomic transitions has been already found in the early high-resolution studies of luminescence from solid Ne [11-13]. It has been shown [11] that the emissions observed stem from the transitions ${ }^{1} P_{1} \rightarrow{ }^{1} S_{0},{ }^{3} P_{1} \rightarrow{ }^{1} S_{0}$, and ${ }^{3} P_{2} \rightarrow{ }^{1} S_{0}$. The spectral features related to the surface and to the bulk were distinguished by their dependence on sample thickness and exciting electron energy [13]. The narrow emission lines were supposed [14] to stem from the desorbing atoms in the states ${ }^{1} P_{1}$ and ${ }^{3} P_{1}$. The wider bulk emissions matched to the transitions ${ }^{3} P_{1} \rightarrow{ }^{1} S_{0}$, ${ }^{1} P_{1} \rightarrow{ }^{1} S_{0}$ were found to be of a doublet structure [13]. The similar features observed in [14] were inter- 
preted in the frame of the coexistence of exciton self-trapping in a regular lattice and trapping in the vicinity of initial nonequilibrium defects of structure, e.g., trapping near a vacancy. Note that the transformation of defects involving A-STE and mobile thermal vacancies at a «high» temperature of $19 \mathrm{~K}$ close to the melting point was considered in [15].

In order to elucidate the internal structure of A-STE emission bands, special experiments on low temperature irradiation by high- and low-energy electron beams were performed $[16,17]$. In the first case the electrons of superthreshold energy $E_{e}>E_{\mathrm{thr}}$ $\left(E_{\mathrm{thr}}=1 \mathrm{keV}\right)$ produced point defects (Frenkel pairs) during excitation via the knock-on mechanism. Based on the dose dependence, the defect related spectral features were clearly identified. For comparison purpose the same luminescence spectra and their dose behavior were studied under irradiation by an electron beam of subthreshold energy $E_{e}<E_{\mathrm{thr}}$, i.e., by electrons of energy deficient to move a lattice atom from the substitutional site to an interstitional one via the knock-on mechanism. A striking resemblance found in the dose behavior - the increase in the intensity of the defect related components, has given conclusive evidence of the electronically induced point defect formation and their accumulation in the lattice of $\mathrm{Ne}$ $[16,17]$. It has been suggested that the self-trapping of excitons into A-STE states is a stimulating factor. However, direct proof of the excitonic mechanism requires state-selective experiments. Synchrotron radiation providing intense vacuum ultraviolet (VUV) light tunable through the exciton absorption range is best suited to this kind of experiments.

The paper reports the results on synchrotron radiation study of permanent lattice defect formation in solid Ne via the excitonic mechanism. The experimental findings are compared with the results of recent molecular dynamics studies of the evolution of the self-trapped excitons into the permanent lattice defects $[18,19]$.

\section{Experimental}

The selective excitation experiments were carried out at the SUPERLUMI station at the Hamburger Synchrotronstrahlungslabor HASYLAB. Since we are interested in a control of the lattice permanent defect creation induced by excitation of electronic subsystem a quality of the samples under study (concentration of initial defects and impurities) is of critical importance. The samples were grown in a special closed cell mounted on a He-flow cryostat holder in ultrahigh vacuum (UHV) environment $\left(10^{-10} \mathrm{mbar}\right)$. The high purity Ne gas $(99,998 \%)$ was used, and the gas handling system was operated under UHV conditions.
The samples were condensed from the vapor phase at elevated temperatures under isobaric conditions $(P=$ $=10 \mathrm{~Pa}$ ). The initial temperature of $18 \mathrm{~K}$ was fixed, whereupon the sample was cooled down to $5 \mathrm{~K}$ with a constant rate of $0.1 \mathrm{~K} / \mathrm{s}$. On the sample preparation the cell was removed and the free-standing crystal was studied. The high quality optically transparent polycrystalline sample of $1 \mathrm{~mm}$ thickness was subjected then to a long-duration exposure to the VUV light. Selective photon excitation was performed with a band pass of $\Delta \lambda=0.25 \mathrm{~nm}$. The excitation energy of $20 \mathrm{eV}$ was chosen in the range of $n=2$ term of the excitonic series. The luminescence analysis was carried out using a $1 \mathrm{~m}$ near-normal incidence monochromator equipped with a position-sensitive detector. The spectra were recorded with spectral resolution $(\lambda / \Delta \lambda)$ of 750 and 1500 . The resonance lines of gaseous Ne were used for the energy calibration. The dose dependence of the luminescence intensity spectral distribution was measured, and the influence of the annealing at $11.5 \mathrm{~K}$ on the spectral features was examined.

\section{Results and discussion}

The typical photoemission spectra of self-trapped excitons in solid Ne taken with different resolution are presented in Fig. 1. Spectrum 2 recorded with the spectral resolution of 1500 shows a number of overlapping spectral peaks. Weak wide band $W$ at the low energy side is known as a vibrationally hot luminescence

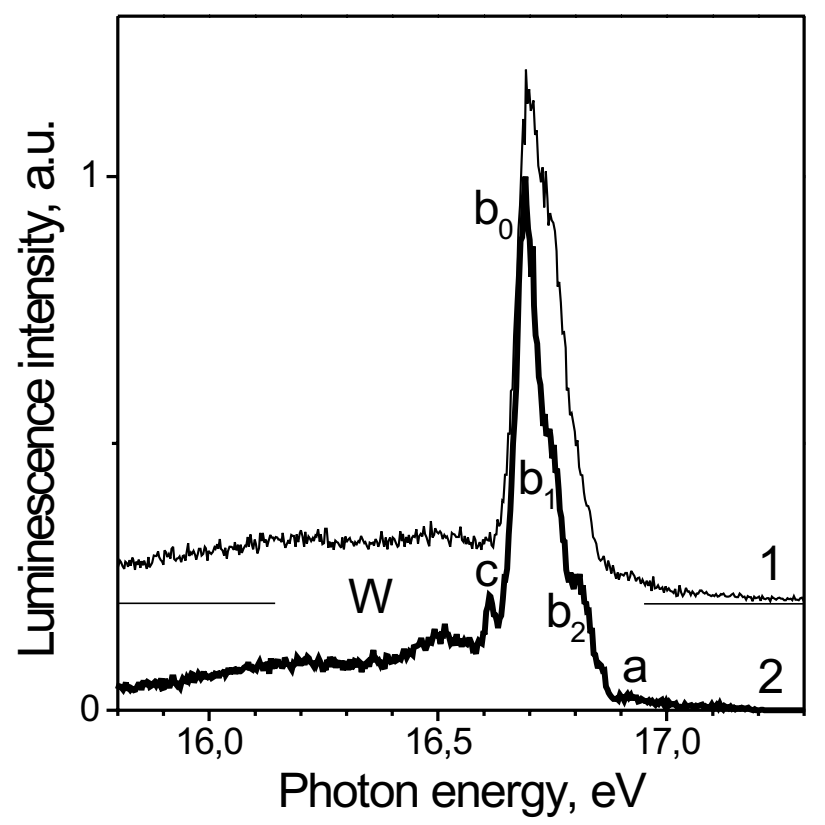

Fig. 1. STE luminescence of solid Ne excited with $20 \mathrm{eV}$ (curve 1) and with $22 \mathrm{eV}$ (curve 2) photons. Curve 2 was recorded with a higher resolution $(\lambda / \Delta \lambda=1500)$. The spectra were measured at $T=5 \mathrm{~K}$. 
of M-STE in solid Ne (see, e.g., $[11,14]$ ). The contributions to the $W$ band from M-STE in the bulk and those at the surface were discriminated in [20]. It is very likely that the desorption of $\mathrm{Ne}_{2}^{*}$ observed in $[21,22]$ contributes the $W$ band too. However, this presumption needs a special check. The most intense structured composite of bands is the A-STE emission in the range $16.6-17 \mathrm{eV}$. The experiments with highly effective excitation of solid Ne by an electron beam $[11,13,14,16,17]$ made it possible to observe the luminescence from three atomic states ${ }^{1} P_{1},{ }^{3} P_{1}$, and ${ }^{3} P_{2}-$ $a, b$ and $c$ bands by the notations given in [16]. All bands have an internal structure with the components of different origin. The narrow $\ll 0 »$ peaks coinciding with the transitions ${ }^{1} P_{1} \rightarrow{ }^{1} S_{0}$ and ${ }^{3} P_{1} \rightarrow{ }^{1} S_{0}$ in the gas phase in energy were identified as emissions from the desorbing atoms in the excited states [14]. The components labeled «1» and «2» which fall into pairs with a splitting close to the spin-orbit splitting, stem from the A-STE centers in the bulk. The «1» components were found to be related to the lattice defects, whereas the $\langle 2 »-$-components were associated with the A-STE in the regular lattice $[14,16,17]$. Several features were assigned to the A-STE at the surface. In the experiments [23] performed with tunable synchrotron radiation the authors were able to isolate some of the peaks by choosing the appropriate excitation energy and time gating. The relevant peak positions and their assignments are listed in the Table.

The intention of the present study was to measure the dose dependence of the intensity distribution in the luminescence spectra of solid Ne under the selective excitation in the range of free excitons. We tuned the excitation energy to the band of exciton absorp-
Positions at $T=5 \mathrm{~K}, \mathrm{FWHM}$, and assignments of the A-STE luminescence components in the range of resonance transitions

\begin{tabular}{c|c|c|c}
\hline \hline Band & Position, eV & $\begin{array}{c}\text { FWHM, } \\
\mathrm{meV}\end{array}$ & Assignment \\
\hline \hline$b_{0}$ & 16.671 & - & ${ }^{3} P_{1}$ (desorbing atom) \\
$b_{1}{ }^{*}$ & $16.746 \pm 0.002$ & $48 \pm 5$ & ${ }^{3} P_{1}$ (defect related A-STE) \\
$b_{2}{ }^{*}$ & $16.807 \pm 0.002$ & $48 \pm 6$ & ${ }^{3} P_{1}$ (A-STE in the bulk) \\
$a_{0}$ & 16.848 & - & ${ }^{1} P_{1}$ (desorbing atom) \\
$a_{1}{ }^{* *}$ & $16.931 \pm 0.005$ & $50 \pm 5$ & ${ }^{1} P_{1}$ (defect related A-STE) \\
$a_{2}{ }^{* *}$ & $16.992 \pm 0.01$ & $45 \pm 5$ & ${ }^{1} P_{1}$ (A-STE in the bulk) \\
\hline \hline
\end{tabular}

Comment. Position and in full width at half maximum $($ FWHM) are given by the data in [23] $(*)$ and [14] $(* *)$.

tion $n=2 \Gamma(3 / 2)$. Due to the low absorption coefficient the exciting light penetrated deep inside the sample and the A-STE centers were efficiently formed in the bulk. The temporal behavior of the luminescence was monitored at $5 \mathrm{~K}$ to rule out thermally stimulated processes. The sequence of spectra was measured during the exposure to VUV light. The most intense $b$ band was analyzed. The initial intensity distribution is shown in Fig. 2, $a$, the final one - in Fig. 2,c. The positions and the widths of the components were obtained from the numerical fit of the band contour assuming the Gaussian shape of the components. The fitting was made taking into account the overlapping of the A-STE emission with a high-energy tail of the

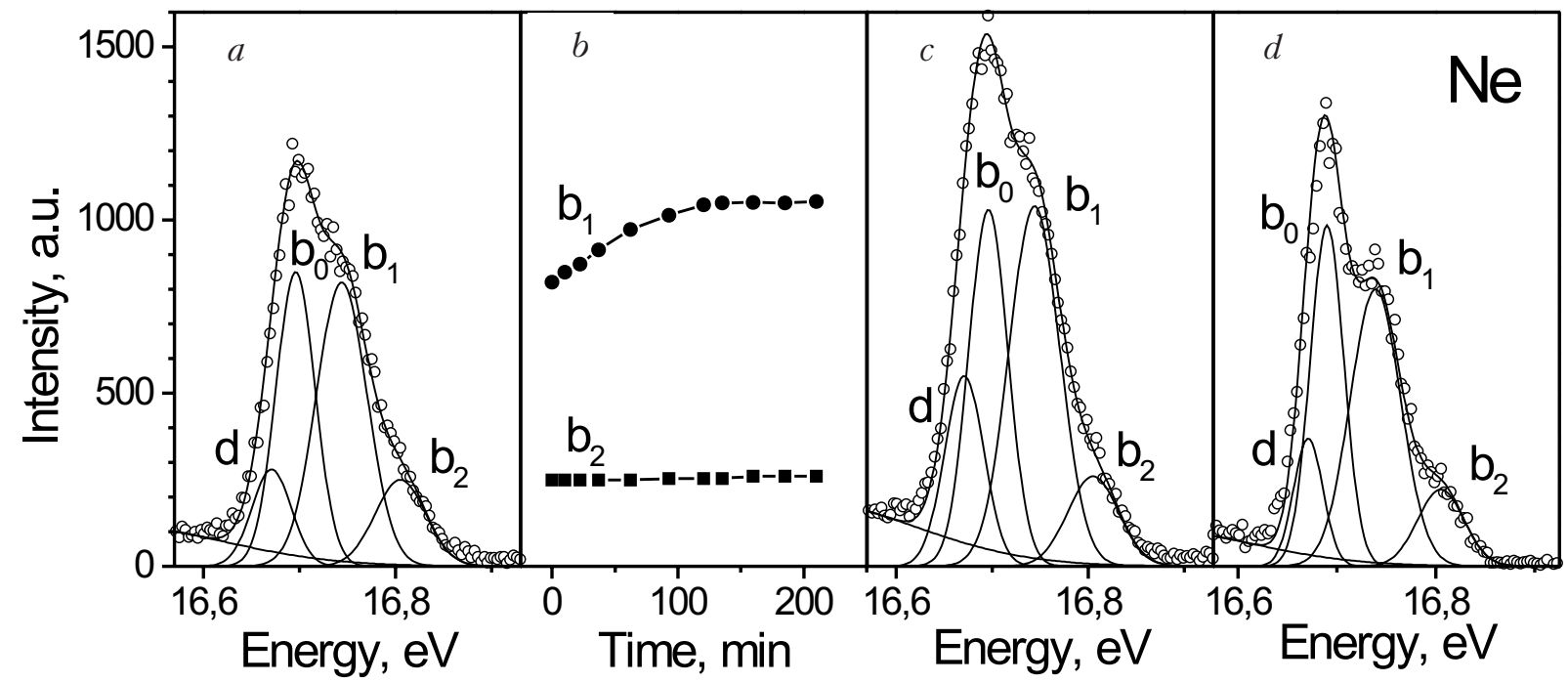

Fig. 2. Photoluminescence of solid Ne in the range of ${ }^{3} P_{1} \rightarrow{ }^{1} S_{0}$ transition ( $b$ band): $(a$ ) and $(c)-$ the evolution of the $b$ band upon irradiation with $20 \mathrm{eV}$ photons, $(b)$ - the dose dependence of the $b_{1}$ and $b_{2}$ components, $(d)-$ the spectrum recorded after the sample annealing at $T=11.5 \mathrm{~K}$. The spectra were recorded at $T=5 \mathrm{~K}$. 
M-STE luminescence shown in Fig. 2. The result of this treatment for the $b_{1}$ and $b_{2}$ components is shown in Fig. 2, $b$ as the corresponding points of the curves. Despite the fact that the $n=2 \Gamma(3 / 2)$ excitons are formed predominantly deep in the bulk, they reach the surface and then are trapped into the A-STE configuration with a subsequent desorption of the excited atom. The appearance of the $b_{0}$ component in the A-STE emission is in line with the results on the partial yield of the Ne atoms in the ${ }^{3} P_{1}$ state [24]. The desorption is due to the microcavity (bubble) formation around the A-STE as a result of the prevailing repulsive interaction between the excited electron and surrounding atoms because of the negative electron affinity of solid Ne [1]. This so-called «cavity ejection» mechanism was studied in detail (see [2] and references therein). The width of the $b_{0}$ component related to the desorbing atoms is resolution limited. The positions and the widths of the $b_{1}$ and $b_{2}$ components are in perfect agreement with the data given in [23]. These bulk components have analogs in the matrix-isolated Ar center spectra [17]. The $d$ component may be tentatively assigned to the transition from the metastable ${ }^{3} P_{2}$ state at defect sites or near the surface. Note, the transition from this state is strictly forbidden in a regular lattice. To the best of our knowledge the geometrical configuration of the centers emitting peaks in the range of ${ }^{3} P_{2} \rightarrow{ }^{1} S_{0}$ transition has not been fully ascertained. Because of the long lifetime of this state and negative electron affinity the chance for the atom to desorb prior the radiation is very high [25]. For this reason the assignment of some emission lines as emissions from the surface centers in the ${ }^{3} P_{2}$ state seems to be very unlikely. The efficient excitation of this emission in the range below the lowest bulk exciton band may also be due to excitation of some centers at defect sites because the defect levels could be expected to occur quite close to the surface exciton band. Note that the $d$ component has an analog in the matrix-isolated center spectra in the range of the low concentrations [17]. The width narrowing and the decrease in intensity observed for the $d$ component upon annealing (see Fig. 2, $d$ ) seems to support the assignment of the $d$ component as being related to the defect sites.

In order to get a direct proof of the permanent lattice defect formation in the initially perfect lattice via the excitonic mechanism, let us consider the features in the range of resonance transition ${ }^{3} P_{1} \rightarrow{ }^{1} S_{0}$. It is just a transformation of the most intense $b$ band on exposure to VUV photons in the range of the second term of the excitonic series well below the band gap energy. The shape of the $b$ band after the cycle of irradiation is shown in Fig. 2,c. The pronounced enhance- ment of the defect related components $b_{1}$ and $d$ was found. As for the $b_{2}$ component associated with the A-STE centers in the regular lattice, it remained unchanged. Figure 2,b depicts the dose dependence of the $b_{1}$ and $b_{2}$ component intensity. The exposure time not corrected for the synchrotron radiation pulse duration is in fact a real time of the measurements. The radiation-induced enhancement of the defect components clearly indicates the formation and accumulation of the permanent lattice defects in solid Ne generated by the self-trapping of excitons into the A-STE states. The 30 min annealing of the samples at $T=11.5 \mathrm{~K}$ caused a decrease in the intensity of the defect related components. The intensities of the $b_{1}$ and $b_{2}$ components were restored. The results obtained in the present experiments with selective excitation accord well with the results of the experiments performed with electron beams [17]. Note, that in these experiments the similar dose dependence was observed for the components of the transitions ${ }^{1} P_{1} \rightarrow{ }^{1} S_{0}$ and ${ }^{3} P_{1} \rightarrow{ }^{1} S_{0}$ (see Table) dipole allowed in a cubic lattice. The similar dose dependences of the A-STE luminescence components found in the cases of superthreshold and subthreshold excitation by electron beams as well as selective excitation into excitonic states provide a convincing proof of the excitonic mechanism of the point lattice defect formation.

The recent excited state molecular dynamics studies $[18,19]$ of a possible evolution of the A-STE states into stable lattice defects suggest a microscopic model of conversion from the A-STE to Frenkel pairs. The elastic deformation followed by the primary bubble formation because of the prevailing repulsive forces between the excited atomic center and surrounding atoms takes place within a very short time. It is interesting that an ultrafast expansion of the bubble was observed upon excitation of the Rydberg orbital of the NO impurity center in solid Ne [26]. Such primary bubbles with elastic deformation collapse once the electronic subsystem returns to the ground state. High local elastic stresses in the lattice can induce some plastic deformation during the lifetime of the excited state. Several cases of possible plastic deformation were examined. It was calculated that the energy levels of some of the bubbles accompanied with a plastic deformation are at energies lower than that of the primary bubble. The lowest energies were found for a bubble with two vacancies in the first atomic shell. It was revealed that the second-nearest neighboring vacancy-interstitial pairs are formed and remain stable in the lattice after exciton annihilation. The estimated differences in energies of radiative transitions of the atomic centers in the primary bubble and in the bubble with plastic deformation $54-66 \mathrm{meV}$ are in perfect 
agreement with the experimental value $60 \mathrm{meV}$ obtained in the present study and with the results given in [14,23] for the ${ }^{3} P_{1} \rightarrow{ }^{1} S_{0}$ and ${ }^{1} P_{1} \rightarrow{ }^{1} S_{0}$ transitions (see the Table). A quite similar process of electronically induced defect formation upon excitation of the Ar atoms in the Neon matrix was observed experimentally [4] and considered theoretically [18]. To some extent the considered mechanism of lattice defect formation resembles that in alkali halides, where the Frenkel pair formation is induced by the repulsive interaction between the excited electron of the STE and core electrons of the molecular ion pairs [1].

\section{Acknowledgment}

It is a pleasure to thank Prof. A.K. Song for fruitful discussions. The authors (E.S. and A.O.) gratefully acknowledge the support by DFG.

1. K.S. Song and R.T. Williams, Self-Trapped Excitons, Springer-Verlag, Berlin (1996).

2. G. Zimmerer, Nucl. Instrum. Methods. Phys. Res. B91, 601 (1994).

3. I.Ya. Fugol', Adv. Phys. 37, 1 (1988).

4. E.V. Savchenko, A.N. Ogurtsov, and O.N. Grigorashchenko, Solid State Phys. 40, 903 (1998).

5. P. Feulner and D. Menzel, in: Laser Spectroscopy and Photochemistry on Metal Surfaces, World Scientific, Singapore (1995), p. 627.

6. M.A. Elango, Elementary Inelastic Radiation-Induced Processes, American Institute of Physics, New York (1991).

7. N. Itoh and A.M. Stoneham, Radiat. Eff. Def. Solids 155, 277 (2001).

8. N. Schwentner, E.-E. Koch, and J. Jortner, Electronic Excitation in Condensed Rare Gases, Springer Tracts Mod. Phys., V. 107, Springer-Verlag, Berlin (1985).

9. I.Ya. Fugol', Adv. Phys. 27, 1 (1978).

10. G. Zimmerer, in: Excited-State Spectroscopy in Solids, Bologna, Corso (1987), p. 37.
11. I.Ya. Fugol', E.V. Savchenko, and A.G. Belov, Sov. Phys. JETP Lett. 16, 245 (1972).

12. E. Schuberth and M. Creuzberg, Phys. Status Solidi B71, 797 (1975).

13. O.N. Grigorashchenko, Yu.I. Rybalko, E.V. Savchenko, and I.Ya. Fugol', Fiz. Nizk. Temp. 8, 886 (1982) [Sov. J. Low Temp. Phys. 8, 447 (1982)].

14. F. Coletti, J.M. Debever, and G. Zimmerer, J. Chem. Phys. 83, 49 (1985).

15. T. Suemoto and H. Kanzaki, J. Phys. Soc. Jpn. 49, 1039 (1980).

16. E.V. Savchenko, Yu.I. Rybalko, and I.Ya. Fugol', JETP Lett. 42, 260 (1985).

17. E.V. Savchenko, Yu.I. Rybalko, and I.Ya. Fugol', Fiz. Nizk. Temp. 14, 349 (1988) [Sov. J. Low Temp. Phys. 14, 220 (1988)].

18. Chun-rong $\mathrm{Fu}$ and K.S. Song, J. Phys.: Condens. Matter 9, 9785 (1997).

19. K.S. Song and Chun-rong Fu, J. Low Temp. Phys. 111, 645 (1998).

20. R. Gaethke, P. Gurtler, R. Kink, E. Roick, and G. Zimmerer, Phys. Status Solidi B124, 335 (1984).

21. E.V. Savchenko, T. Hirayama, A. Hayama, T. Koike, T. Kuninobu, I. Arakawa, K. Mitsuke, and M. Sakurai, Surf. Sci. 390, 261 (1997).

22. T. Hirayama, A. Hayama, T. Adachi, I. Arakawa, and M. Sakurai, Phys. Rev. B63, 5407 (2001).

23. W. Laash, H. Hagedorn, T. Kloiber, and G. Zimmerer, Phys. Status Solidi B158, 753 (1990).

24. T. Kloiber and G. Zimmerer, Phys. Scrypta 41, 962 (1990).

25. I. Arakawa, T. Adachi, T. Hirayama, and M. Sakurai, Fiz. Nizk. Temp. 29, 342 (2003) [Low Temp. Phys. 29, №3 (2003)].

26. F. Vigliotti, L. Bonacina, M. Chergui, G. Rojas-Lorenzo, and J. Rubayo-Soneira, Chem. Phys. Lett. 362, 31 (2002). 